\title{
Communication
}

\section{Short Reviews of the Francisco Bulnes's Mathematical Research Theory}

\author{
Helen Thrift ${ }^{1}$, Michelle Musk ${ }^{2}$ \\ ${ }^{1}$ Department of Physics, Edinburg University, Edinburg, United Kingdom \\ ${ }^{2}$ Department on Biographical Research, International Biographical Centre, Cambridge, United Kingdom \\ Email address: \\ Thrift.helen@ed.as.uk (H. Thrift),musk@ibc.com (M. Musk)
}

\section{To cite this article:}

Helen Thrift, Michelle Musk. Short Reviews of the Francisco Bulnes's Mathematical Research Theory. International Journal of Systems Science and Applied Mathematics. Vol. 4, No. 1, 2019, pp. 13-17. doi: 10.11648/j.ijssam.20190401.12

Received: August 23, 2019; Accepted: September 5, 2019; Published: September 19, 2019

\begin{abstract}
In this short communication, we revised a mathematical theory on scientific research created by doctor Francisco Bulnes, to be applied in the modern research, which proves, and explains the unity that must exist in applied and theoretical research to obtain true results considering the necessary and sufficient contents in a scientific research. Also because of this theory is deduced a mathematical theory of engineering, that is to say, a mathematical theory of the imagery and creativity necessaries in engineering research. Banach spaces are created and used to develop the doctor Bulnes's theory, which determines a methodology of scientific research based in mathematics, or at least whose rector axis are the mathematics. Here the difference of knowledge levels are showed in evident way, classifying the research in three levels, where the fundamental level is the creation of a good and consistent theory. After in second plane will come the application of this theory in the inductive or applied sciences level creating a sub-theory. Finally, in the level more applicative or technological, we will have the prototypes, which will derive in the technological products giving solution to society necessities. One principal result of this theory is that the engineering does technology on energy.
\end{abstract}

Keywords: Bulnes's Research Theory, Knowledge Space, Optimal Prototype, Prototypes, Technologies, Theories,

True Propositions Space, Sub-theories

\section{Introduction}

A new research theory has been created in the last 20 years by the Dr. Francisco Bulnes, which has been working in mathematical theories very enclosed with the quantum field theories, theoretical physics focused in the gravity and nanotechnology in nanomaterials and nanomedicine [1-3]. In the last years also have had development the quantum electronics, where are searching advances in quantum communication, artificial intelligence, and advanced energy transducers. Developments in photonics and spintronics until does little, are determinant to produce advanced technology to quantum electronics in diverse processes where are required other principles in the science, new physics and more fine re-interpretations of the topologies and modern geometries. The prospective in the use of the energy process of the electrons conduct to major developments of the advanced electronics considering no only their photon but also the manager of their spin giving as result the spintronics [4]. This last also consider to the electron as the fundamental particle in all the processes of transference and information to the manager of other particles considering basal and transitory states of the electron through technologies as are the dots, magnetons or spin-transistors [5-9] (see figure 1).

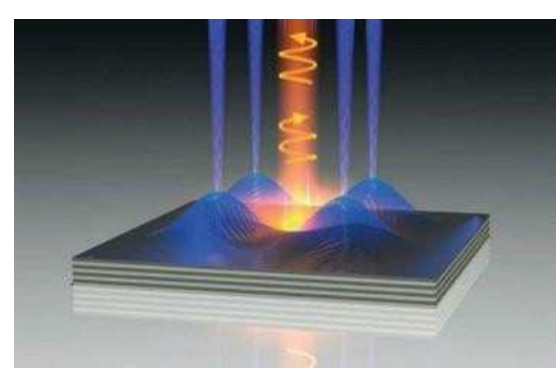

A) 


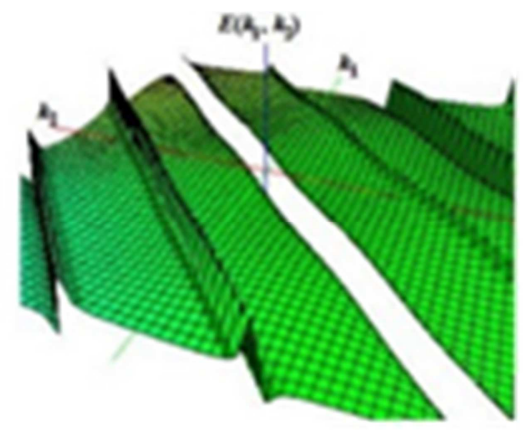

B)

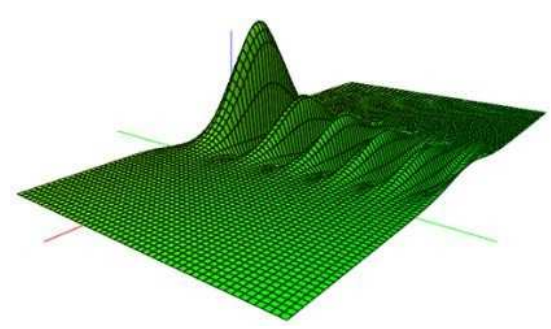

C)

Figure 1. A). Liquid light to help to the development of future advanced electronics in the threshold of the spintronics in the transducers design. B). Direct sum of states to establish the curvature energy by the field ramification [5-7]. C). Surface of energy to a metallic structure whose energy and prohibit trenches of energy in the solid were obtained applying $a$ elementary electric charge that produce a planar constant superconductivity $[8,9]$.

In the 90's decade, the Dr. Bulnes creates a mathematical theory on the engineering called FET (Formal Engineering Theory), which developed his research in his trips in the south East of Mexico and Cuba country [10]. After, and adding tools of Lie groups and their algebras, likewise as electronics theory, he creates a research theory which he called modern research, since incorporates the nanotechnology and the quantum field theory. After of various years in a visit to United Kingdom, he gives a seminar of three days in the London city with the purpose of share this new view of the scientific research focused to new knowledge areas. In addition, his research method has been applicable to the reviewing of the British journals indexed in SCOPUS [11-13] (See figure 2).
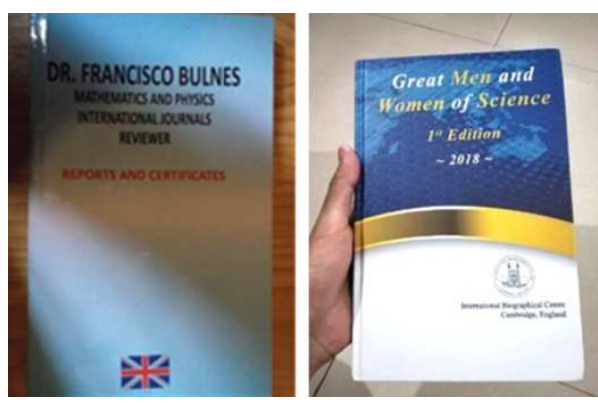

Figure 2. Book on reviewer record and reports of papers published and revised in British journals of mathematics, physics and technology. This book reported more than 100 papers revised All contents have registers in Publons [12, 13]. B) Biography of outstanding scientist registered in United Kingdom Record Guinness. Book published by Melrose Place Press, Cambridge, UK [11]. The Editor of this book is Sara Rains.

\section{The Fundamental Bulnes's Theorem on Modern Research}

Theorem (F. Bulnes) 2. 1. [14] A). $X_{L A B}$ is isometric to $E_{\text {FISMAT }}$ B). $\Phi_{\alpha}\left(t_{\beta}\right)=X_{L A B}\left(\Phi_{\alpha}\right)$.

Proof. [15].

A grosso modus, the theorem says to the A), that $X_{L A B}$, and $E_{\text {FISMAT }}$ follow the same metric of acknowledge (in times and advances in research). An example has been the research line given for curvature sensors.

The incise B), says that the obtained results in laboratory must be congruent with the obtained results in the theory. Furthermore, establishes an identity inside the knowledge creation (epistemology of the science) an equivalence inescapable inside the knowledge creation, because much knowledge can be produced with improves of the existing technologies and for other way, the experimental research must be founded in the theory of the knowledge. Both are measurable in duality.

This generates certainty and consistence of the research prospective [16].

Such as affirms, the doctor Francisco Bulnes, the field observables are elements of $H^{*}$ where $H^{*}$ is the 2-cuadrable measures energy signals space ${ }^{1}$.

In the modern research, the managing of the time is fundamental. Likewise, are established priorities inside the research classifying to the research projects and lines respect to the time as projects of large, average and short scope.

The research time can be designed as time series, that is to say, to risk the objectives for steps. This obeys to the proper research evolution. What defines that evolution? The research development. How to establish that this research development is the most optimal? This obeys to the efficient causality principle in the Universe, which affirms that any optimal effect or efficient effect must come from an efficient cause. Then is necessary an optimal design of the cause. Here is where starts.

The time is the shortest length between cause and effect.

Proposition (F. Bulnes) 2. 1. The efficient cause inside the research theory comes from a good theory.

Proof. See [17].

All application will be corollaries of this good theory. Then the theoretical knowledge is fundamental.

However, also we have through an universal principle that the cause is included in each one of the applications, because effect is included in the cause and cause also is included in the effects. The great mathematician Hilbert (the Modern Euclides) says:

"The best application of the acquired acknowledge is a good theory".

In the research method for fundaments, the causality is given in the design of a Th $\Sigma$ consolidated through publications in indexed and arbitrated journals and reviewed

1 We consider the mapping $T: H \rightarrow H^{*}$ with rule of correspondence $\mathrm{f}(\mathrm{x}) \mapsto \mathrm{T}(\mathrm{f}(\mathrm{x}))=\mu(\mathrm{x}) \quad[18,19]$. 
by academic pairs. Likewise, the research ethic is normed for the rules established to accredit the scientific work. Then we can to define:

Def. (F. Bulnes) 2. 1. The ethic in the research is the moral quality or harmony between the individual in relation to a scientific work.

\section{Research in Engineering}

Really, there are three fundamental aspects to the formal engineering theory (FET):

a) The engineering is the creation of the technologies in base of managing of the energy.

b) The engineering is the final product of the fundamental sciences: Mathematics and Physics.

c) The engineering is the constant searching of the improvements in process and developments in technology.

Likewise, we consider $E$ the set of mathematics and physics knowledge, $\Phi$ the set of mathematics and physics true knowledge. Th the theories space that can generate and $I_{\sigma}$ transducers research. Who is $I_{\sigma}\left(E_{\text {FISMAT }}\right) \rightarrow T h \Sigma^{0} \rightarrow 0$ ?

What is necessary to know in the ambit of the mathematics and physics to create a transducer? Here is where enter the space $I_{\sigma}\left(E_{F I S M A T}\right)$. Likewise we have for one side, in the mathematics part, the functional analysis, the differential equations and geometry.

For other side, in the physics part, the physical systems dynamics, the electromagnetism, optics, acoustics, fluid mechanics, thermodynamics, pneumatics and modern physics. Then in the inferior level of $I_{\sigma}\left(E_{\text {FISMAT }}\right)$ is required a more specialised knowledge of mathematics and physics: solid state, circuits, servo-systems, control theory, stability theory, opto-electronics, electronics, semiconductors and superconductors, robust programming.

We fix as research problem, some problem related to the design, creation and functioning of a transducer. Then in the context of the technological prototypes are considered the following commutative diagram [20,21]:

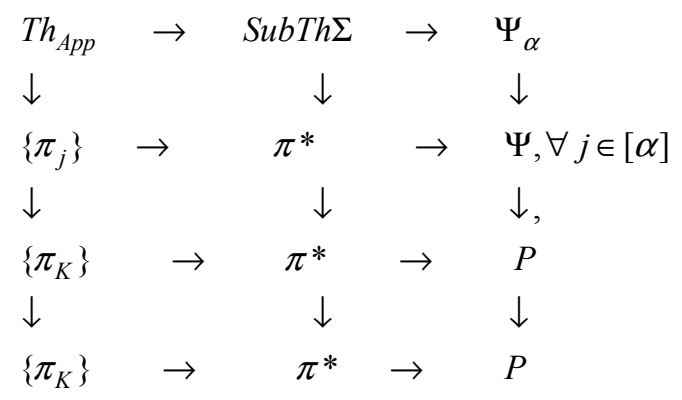

where $\Psi_{\alpha}$ is the models algebra of the class $\alpha$ the set $\left\{\pi_{j}\right\}$ is a set of prototypes which are choice the $\pi_{K}$ to develop through the application of a theory $T h_{A p p} \rightarrow \operatorname{SubTh\Sigma }$ the optimal prototype $\pi^{*}$.

This optimal final prototype is a product if can give a solution to a society problem: $P \rightarrow 0$.

Example 3. 1. We want manufacture a special rod with maximum resistance tension with minimal displacements $u(t)$ in their inner. The special rod could be a technology $t_{\sigma}$ on the existing, which we call $t_{\alpha}$ belonging to the class $\alpha$ of beams and pillars. First in the level of theoretical research the Bulnes's research method can be described to this research type as $[17,21]$ (figure 3 ):

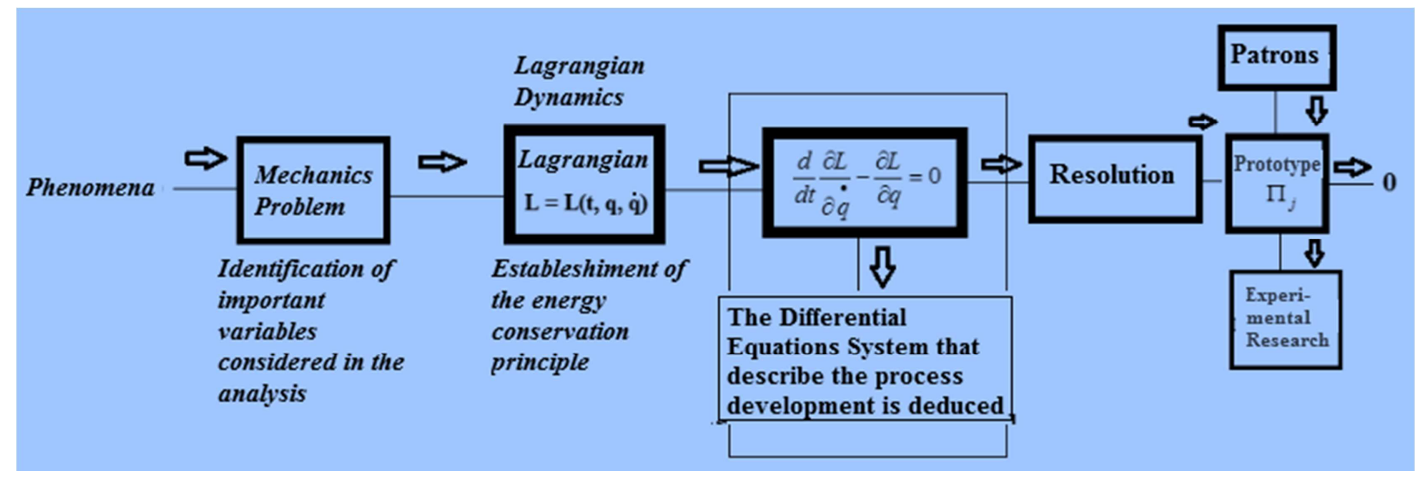

Figure 3. Blocks diagram of research in physical dynamics.

After in the prototype level $T h_{A p p} \rightarrow \operatorname{SubTh\Sigma }$ we have their evaluation given for the value of the Bulnes's integral of execution of the technology:

$$
\operatorname{Exe}\left(t_{\sigma}\right)=\int_{\Phi \alpha} \varphi_{\gamma} \Phi_{\alpha} A\left(\Phi_{\alpha}\left(E_{F I S M A T}\right)\right) \mu\left(\Phi_{\sigma} E\right)
$$

$$
\begin{array}{r}
\|u\|_{\text {Patron }}^{2}=\int_{0}^{l} G S(t) u^{\prime 2}(t) d t \\
\leq E S_{1} \int_{0}^{l}\left(u^{2}(t)+u^{\prime 2}(t)\right) d t \\
\leq G S_{1}\|u(t)\|_{1,2}^{2}
\end{array}
$$

which will derive in an integral of energy 
where $G$ is Young's modulus, $S(t)$ is the area of the cross-section with $S_{0} \leq S(t) \leq S_{1}$ and $u(t)$ is the displacement of the cross-section of the rod at point $t$ in the longitudinal direction. We have supposed that at $t=0$ is fixed, that is to say $u(0)=0$.

\section{Conclusions}

The new proposed method who is author, the Dr. Francisco Bulnes, has as rector axis the mathematics. This is advantage in the research in any area or theme, because obligate to study the things by their essence and fundament. The research in engineering using the research by fundamentals has to do it under levels of the same research scheme established for the method, where are acquaintances three steps or levels in the case of the commutative diagram: the first level is determined for the development of a sub-theory considering the morphism $T h_{A p p} \rightarrow \operatorname{SubTh\Sigma }$. The second level consists in the determining of the optimal prototype under research of a set of prototypes $\left\{\pi_{j}\right\}$. Finally the third level is the solution to a social necessity, $P \rightarrow 0$.

The relevance of this theory will be the optimization of the steps in the research, the certainty of the information sources to the particular scientific research and the invariance of these methods that permit carry to all orders of research as the experimental research.

\section{Nomenclature}

$X_{L A B}-$ Topological vector space of experiments or rehearsals in the technological prototypes. This space has structure as metric space. This is a Hilbert space.

Th $\Sigma$ - Theory of the knowledge $\Sigma$.

$E_{\text {FISMAT }}-$ Set or space of all acknowledges in physics and mathematics. This is the Universe in Formal Engineering theory, and also in modern research theory. This is a Banach space.

$H^{*}$ - Hilbert space of the measures obtained in the instruments, experiments and rehearsals.

$\Phi_{\alpha}-$ Space of true propositions in the class $\alpha$. This is a subset of $E_{\text {FISMAT }}$ This is the fundamental set to start a theory $T h \Sigma$.

$t_{\sigma}-$ Technology of the class $\sigma$. From a point of view of technology theory is a neologism of a given technology. From a point of view of mathematics are points $t_{\sigma}, t_{\rho} \in \mathrm{Gr}_{\lambda}$ of $\mathrm{G}_{\lambda}$ an morphisms group whose automorphisms $\lambda$ are in a topological group $\mathcal{G}$ which could have Lie group structure when we talk of technologies on the energy, that is to say on the Hilbert space structures.

$G$ - Topological group of the operator class of acknowledge.

$\mathrm{Gr}_{\lambda}-$ Group of morphisms whose automorphisms are in the topological group $G$.
$T h_{A p p}-$ Theory obtained through the experimental study and applied science.

$\operatorname{SubTh\Sigma }-$ Sub-theory of the specialised knowledge.

\section{Acknowledgements}

We are very grateful with Prof. Dr, Francisco Bulnes IINAMEI Director and Head of Research Department in Mathematics and Engineering, TESCHA by his material support and facilities to realize this research.

\section{References}

[1] F. Bulnes, "Design of Quantum Gravity Sensor by Curvature Energy and their Encoding", IEEE Proc., SAI 2014, London, UK, 2014, pp. 855-861.

[2] F. Bulnes, Y. Stropovsvky and V. Yermishkin, "Quasi-Relaxation Transforms, Meromorphic Curves and Hereditary Integrals of the Stress-Deformation Tensor to Metallic Specimens," Modern Mechanical Engineering, Vol. 2 No. 3, 2012, pp. 92-105. doi: 10.4236/mme.2012.23012.

[3] F. Bulnes, F. H. Bulnes-González, "Quantum Developments in Nanomedicine: Nanocurative Actions by Soft Photons Sources and their Path Integrals," A. Seifalian, A. de Mel, D. M. Kalaskar (Eds.), Open Central Press, United Kingdom.

[4] Dr. Francisco Bulnes, Editor-in-Chief, Journal on Photonics and Spintronics, Years, 2012-actually http://researchpub.org/journal/jps/editorial\%20board.html.

[5] Bulnes, F. Martínez, I, Mendoza, A. Landa, M., "Design and Development of an Electronic Sensor to Detect and Measure Curvature of Spaces Using Curvature Energy," Journal of Sensor Technology, 2012, 2, pp116-126. http://dx.doi.org/10.4236/jst.2012.23017.

[6] F. Bulnes, I. Martínez, O. Zamudio, Fine Curvature Measurements through Curvature Energy and their Gauging and Sensoring in the Space, Advances in Sensors Reviews 4, (Ed.) Sergey Y. Yurish, IFSA, 2016.

[7] Francisco Bulnes (June 14th 2017). Detection and Measurement of Quantum Gravity by a Curvature Energy Sensor: H-States of Curvature Energy, Perturbation Theory Dimo I. Uzunov, IntechOpen, DOI: 10.5772/68026. Available from:

https://www.intechopen.com/books/recent-studies-in-perturbat ion-theory/detection-and-measurement-of-quantum-gravity-by -a-curvature-energy-sensor-h-states-of-curvature-ener.

[8] F. Bulnes, S. Humeini, Topological Superconductors Characterizing II: Curvature Energy in Hall-Spintronics Developments, SciFed Journal of Spintronics and Quantum Electronics, 1:1, 2018.

[9] Francisco Bulnes (2015). A Lie-QED-Algebra and their Fermionic Fock Space in the Superconducting Phenomena, Selected Topics in Applications of Quantum Mechanics, Prof. Mohammad Reza Pahlavani (Ed.), InTech, DOI: 10.5772/59078. Available from: https://www.intechopen.com/books/selected-topics-in-applicat ions-of-quantum-mechanics/a-lie-qed-algebra-and-their-fermi onic-fock-space-in-the-superconducting-phenomena. 
[10] F. Bulnes, Teoría de Algoritmos para la Maestría de Informática Aplicada, Universidad de las Ciencias Informáticas (UCI), Habana, Cuba, Postdoctoral Project (Aplicaciones de la Teoría Infinita de Lie y sus Aplicaciones a Informática) 2006-2007.

[11] Law, S. N. Musk, M. (2018) Great Men and Women in Science, International Biographical Centre, Cambridge, Sara Rains (Ed.), UK. ISBN: 978-1-903986-44-8 (1st Edition).

[12] Thrift Helen, "DR. FRANCISCO BULNES: Mathematics and Physics International Reviewer" Reports and Certificates Compilation, SCIENCEDOMAIN International, United Kingdom, 2017.

[13] Record Publons: Metrics and Statistics as Reviewer of Dr. Francisco Bulnes, 2018.

[14] Bulnes, F. Stropovsvky, Y. and Rabinovich, I. (2017) Curvature Energy and Their Spectrum in the Spinor-Twistor Framework: Torsion as Indicium of Gravitational Waves. Journal of Modern Physics, 8, 1723-1736. doi: 10.4236/jmp.2017.810101.

[15] Bulnes, F. (2013) Mathematical Nanotechnology: Quantum Field Intentionality. Journal of Applied Mathematics and Physics, 1, 25-44. doi: 10.4236/jamp.2013.15005.

[16] F. Bulnes, "Analysis of Prospective and Development of Effective Technologies through Integral Synergic Operators of the Mechanics," 14a. CCIA-CCIM, ISPJAE, Habana, Cuba, 2008.

[17] Bulnes F., Teoría de la Investigación en Ciencias de la Ingeniería, Editorial Académica Española, 2010.

[18] Simon and M. Reed, "Mathematical methods for physics, Vol. I (functional analysis)," Academic Press. N. Y., 1972.
[19] F. Bulnes, Tratado de Matemáticas Superiores: Análisis de Señales y Sistemas, ETC, Facultad de Ciencias, UNAM, 1998.

[20] F. Bulnes, Zuckerman Functors, Notas del Seminario de Teoría de Representaciones de Grupos de Lie Reductivos Reales, Instituto de Matemáticas, UNAM, 2003.

[21] Bulnes F., El Modelo de Competencias en la Investigación Moderna, EAE, 2018. ISBN: 978-620-2-13528-3.

\section{Biography}

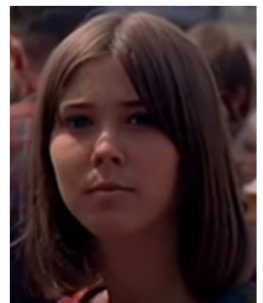

Helen Thrift, Chair Professor Cambridge University. Her research is focused in the historical memory preservation of the academic relationships with other academicians and researchers in any acknowledge area. She has the highest intellect coefficient in South England. She has University, United Kingdom. a doctorate degree in physics by the Edinburg

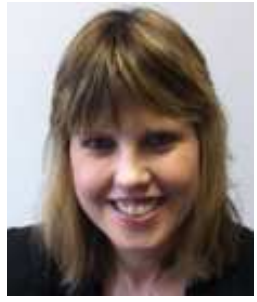

Michelle Musk, Melrose Press administrator with 20 years' experience seeking a new role Littleport, Cambridgeshire, United Kingdom. Administrative Executive of IBC and international travel agent. Actually, she works and lives in Ely, Cambridgeshire, United Kingdom. 